\title{
Markov Chain Monte Carlo simulation of electric vehicle use for network integration studies
}

\author{
Yue Wang ${ }^{\mathrm{a}}$, David Infield ${ }^{\mathrm{b}}$ \\ ${ }^{a}$ Corresponding author, Ellison Building, Northumbria University, Newcastle upon Tyne, NE1 8ST, UK, email: \\ yue5.wang@northumbria.ac.uk; \\ ${ }^{\mathrm{b}}$ Department of Electronic and Electrical Engineering, University of Strathclyde, 204 George Street, Glasgow, G1 1XW, UK, \\ e-mail: david.infield@strath.ac.uk
}

\begin{abstract}
As the penetration of electric vehicles (EVs) increases, their patterns of use need to be well understood for future system planning and operating purposes. Using high resolution data, accurate driving patterns were generated by a Markov Chain Monte Carlo (MCMC) simulation. The simulated driving patterns were then used to undertake an uncertainty analysis on the network impact due to EV charging. Case studies of workplace and domestic uncontrolled charging are investigated. A $99 \%$ confidence interval is adopted to represent the associated uncertainty on the following grid operational metrics: network voltage profile and line thermal performance. In the home charging example, the impact of EVs on the network is compared for weekday and weekend cases under different $\mathrm{EV}$ penetration levels.
\end{abstract}

Keywords-Electric vehicles, Markov Chain, Monte Carlo, multi-place charging, uncertainty.

\section{Introduction}

As the EV penetration level increases, accurate prediction of the associated electricity consumption is required for network side planning, in particular, network asset investment, [1]. The associated uncertainty in load is also of essential importance to the network normal operation since it allows the network operator to leave sufficient margins during the planning stage as well as in operation. The large sample size that is required for the uncertainty investigation justifies the construction of a suitable model for detailed simulation of vehicle use patterns.

Stochastic techniques lend themselves to vehicle use modelling due to the random nature of driving patterns. Monte Carlo simulation, as a stochastic modelling approach, is a popular choice, such as [2], [3], [4], [5], and [6].

Detailed Markov Chain Monte Carlo (MCMC) simulation was used to generate weekly EV patterns in [2] with half hourly resolution. The initial states were defined assuming a Gaussian distribution for EV characterisation, and the subsequent instances were produced based on the Markov Chain transition probabilities using Monte Carlo simulation. One infeasible assumption in this particular simulation was that the driving period per journey was fixed to be 30 minutes, which is due to the lack of self-transition status of driving, and this limits the generation of continuous vehicle driving states.

Iversen et al. also employed Markov Chain models for describing vehicle diurnal driving patterns in [3], including the discrete time Markov model, where the size of the state transition matrix was proportional to the time resolution and vehicle states considered, the continuous time Markov model, where possible parameter reductions can be obtained compared with the discrete model, and the hidden Markov model, which allowed for modelling states that are not directly observed in the data by introducing a new state to the original Markov model. The associated application however was constrained to the two vehicle status of 'driving' and 'not driving' without any charging locations allocated.

Another example of Monte Carlo simulation is presented in [4] where three key variables, consisting of the time of vehicles' arrival and departure time at and from charging locations and the travelled distance in between, were selected from a transportation database for vehicle motion generation. Since the variables were statistically dependent, a copula function was employed to join the univariate distribution functions to build the joint multivariate distribution function for both a single and double journeys, which was then used for the Monte Carlo simulation to model vehicle use patterns.

A Gaussian distribution, non-uniform distribution and conditional Gaussian distribution was assigned in [7] for simulating the arrival time, charging time and departure time, respectively. Similar distributions have also been assumed for EVs' arrival and departure in [8] and [9] respectively. In reality, however, such predefined distributions are sometime unsuitable for vehicle pattern simulation. For example, [10] sampled from a Gaussian distribution function to synthetize the travelled distance, which according to [4] was correlated with and therefore should be determined by the departure time of the commuter.

Reference [1] also proposes a statistical modelling approach to generate daily driving patterns, where the temporal distribution of departure and arrival times and their correlation were modelled first, and the synthetic driving cycles associated with the driving distance distribution were then constructed. The acceleration related variables used for synthetic driving pattern construction in [1] are however unavailable in most of the transportation data, which would limit the application of the method.

A multi-agent system with percolation approach is presented in [11] for simulating EVs' driving patterns, where mobile and static agents are employed and the percolation methodology is used to identify probable locations of $\mathrm{EV}$ charging activities.

It is important that the synthetized vehicle patterns are verified against the original vehicle records, but so far not 
many publications have been found that have undertaken this. The closest are the work presented in [5], where the seasonal charging loads that were generated from various stochastic models were compared with those from the original GPS based vehicle use patterns, and [1], where simulated arrival time distribution is verified against the original real-life measurement. This present paper makes a contribution by providing a detail verification of vehicle characterisation as well as different charging location scenarios.

Following on from the development of synthetic EV driving patterns, these models have been used to analyse the impact of EV charging on the distribution network, such as [6], which estimated the thermal effect of EV charging on transformer aging, and [12], in which the peak load, total loss and voltage violation of the system due to the EV charging load under different year scenarios were investigated.

It is understood that the stochastic nature of EV motion would lead to uncertainties in the EV demand curve and therefore network operational metrics. The uncertainty of EV load due to one million EVs under the scenario of uncontrolled domestic charging was analysed in [4], but only on a nationally aggregated demand scale. This present paper calculates the uncertainties of the EV demand and associated network metrics due to EV patterns using the MCMC simulation. Such uncertainty analyses provide precious information for the network operators and also help with system planning. The other strengths of this work are the high time resolution of ten minutes and the detailed and verified representation of vehicle use.

National transportation statistics such as National Household Travel Survey [13] and Mobility Research Netherlands [14] have been in support of EV investigations in [6] and [4] respectively. The present work utilizes the 2000 UK Time of Use Survey (TUS) data because of its high time resolution and in order to be consistent with the domestic electricity consumption model [15] which was developed based on the same set of data and will be employed here to generate the domestic base load, house by house.

The work presented in this paper utilizes MCMC simulation to generate synthetic EV use patterns based on vehicle movement characterisation, in this case from the TUS data. By using MCMC simulation, the impact of the uncertainty in EV load on the power system is investigated at a distribution network level.

\subsection{Contribution of this work}

The fore-mentioned works have been summarised in Table 1 in terms of 6 model features that the presented work possesses, from which it can be seen that the contributions of this work are fine data resolution, which allows detailed and accurate vehicle movement modelling, verification of vehicle driving patterns, which guarantees the simulation accuracy, and uncertainty analysis of network impact, which is important for grid planning and operation.

The MCMC simulation presented in [16] was rather provisional in the sense that model results were not subject to verification and uncertainty analysis for practical network assessment was not undertaken. The present paper includes both these important elements.

Table 1

Summary of relevant literature works

\begin{tabular}{|l|c|c|c|c|c|c|}
\hline & A & B & C & D & E & F \\
\hline$[1]$ & $\checkmark$ & $\checkmark$ & $\checkmark$ & $\checkmark$ & - & - \\
\hline$[1]$ & x & $\checkmark$ & x & x & $\checkmark$ & x \\
\hline$[3]$ & $\checkmark$ & x & $\checkmark$ & $\checkmark$ & x & x \\
\hline$[4]$ & x & $\checkmark$ & $\checkmark$ & x & x & x \\
\hline$[5]$ & x & $\checkmark$ & $\checkmark$ & $\checkmark$ & x & x \\
\hline$[6]$ & x & - & - & - & $\checkmark$ & x \\
\hline$[7]$ & x & $\checkmark$ & x & - & x & - \\
\hline$[10]$ & x & $\checkmark$ & x & x & - & - \\
\hline$[12]$ & x & $\checkmark$ & x & - & $\checkmark$ & x \\
\hline$[16]$ & $\checkmark$ & $\checkmark$ & $\checkmark$ & x & $\checkmark$ & x \\
\hline This work & $\checkmark$ & $\checkmark$ & $\checkmark$ & $\checkmark$ & $\checkmark$ & $\checkmark$ \\
\hline
\end{tabular}

A: Fine data resolution (less or equal to 10 minute per step)

B: Vehicle status definition

$\mathrm{C}$ : Vehicle movement simulation

D: Vehicle use pattern verification

E: Detailed network impact analyses considering charging location

F: Uncertainty analysis of detailed network impact

$\checkmark$ : model feature is included in a suitable manner

$\mathbf{x}$ : model feature not included

-: not relevant

The work in [2] was simplified by assigning a fixed driving period of 30 minutes per journey in the Markov Chain transition model construction, which has a completely different driving period distribution from the TUS data, as will be presented later in this paper. The vehicle movement modelling using this assumption in [2] would therefore be inaccurate. This present work contributes to improve the Markov Chain model by adding a self-transition of driving to the state transition diagram which was absent in the work of [2], and the finer data resolution defined by the TUS data used here is capable of capturing more accurate vehicle movement.

\section{Time of use survey (TUS) data}

The UK 2000 time of use survey (TUS) was carried out on domestic activities for both weekdays and weekends including the use of privately owned vehicles. Each survey was on a 24-hour basis with 10-minute resolution, starting at $4.00 \mathrm{am}$ and ending at 3.50am the next day, since activities reached a minimum around this time of day [17]. The vehicle driving related survey diaries were chosen for the analyses in this work, and the vehicle status for such diaries were classified into four distinct states: namely 'driving'; 'parking at home'; 'parking at workplace'; and 'parking at commercial areas'. The vehicles' departure and arrival activities and individual journey time could then be subsequently obtained for the selected database. It should be noted that a further process of removing any diaries with a daily journey longer than 180 minutes was undertaken to reflect the feasible electric range of EVs in accord with the EV specification in Section 4. The number of diaries from the processed TUS data is 1476 and 2642 for the weekday and weekend data, respectively. 


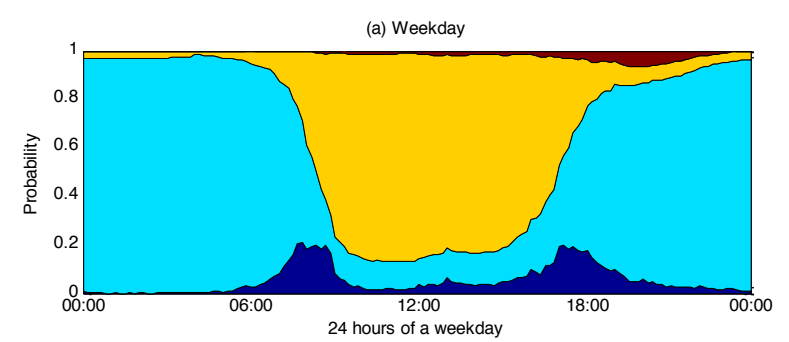

(b) Weekend

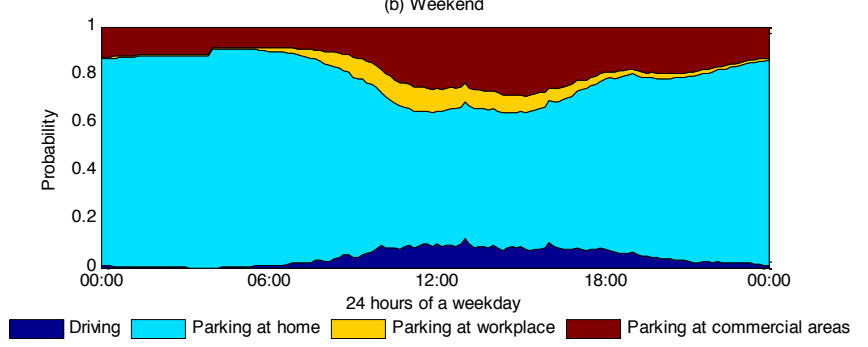

Fig. 1. Vehicle state proportions during weekdays and weekend
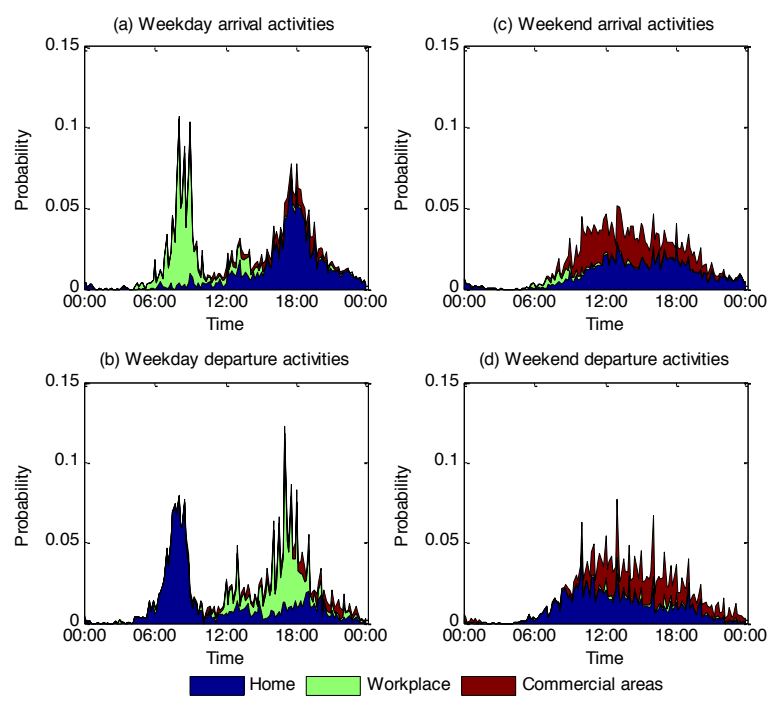

Fig. 2. Vehicles' arrival and departure probability during weekdays and weekend

The statistical characteristics of the selected TUS data that involve vehicle driving are illustrated in Figs. 1 to 4 for both the weekday and weekend case. In both cases the vehicle activities are configured based on the assumption of a periodically stationary daily cycle from $4 \mathrm{am}$ to $3.50 \mathrm{am}$ the next day due to the minimal activities at this period, which is verified by the smooth transition at this time of day as illustrated in Fig. 1. A morning and evening weekday driving peak can be overserved in Fig. 1(a), which can be confirmed as for commuting purpose by referring to the associated arrival and departure activities from Fig. 2(a), where the probability value is obtained by dividing the associated activities by the total number of diaries considered. The weekend TUS data, illustrated in Fig. 1(b) and 2(b), show significant differences from the weekday case in terms of driving patterns and parking locations.

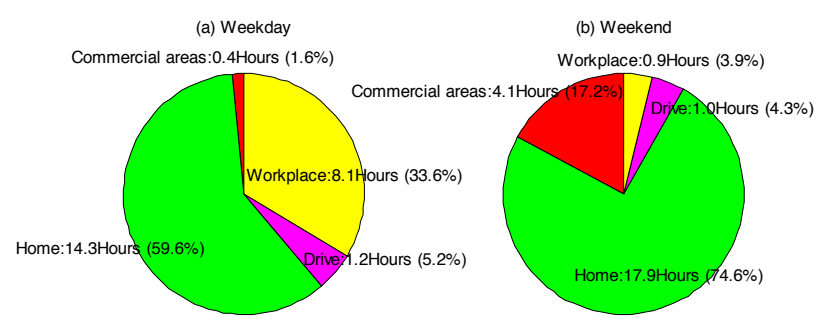

Fig. 3. Averaged time spent in different vehicle states for weekdays and weekend

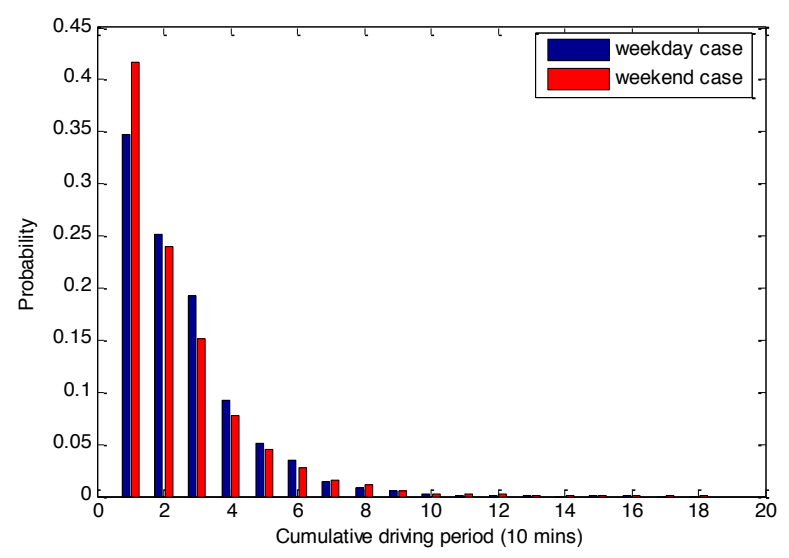

Fig. 4. PDF of vehicle driving period for weekday and weekend

It is also obvious from Fig. 1(a) that the majority of weekday vehicles park at the workplace during daytime and at home during night time, indicating the potential charging time and locations for the EVs. The average weekday proportion for vehicles parking at home $(59.6 \%)$ and workplace $(33.6 \%)$, as shown in Fig. 3(a), further emphasizes the significance of these two locations for providing EV options for grid service provision via charging and discharging under a smart grid environment. Special attention is drawn to the workplace and commercial areas related activities, the dominance of which changes dramatically between the weekday and weekend cases. The average time for the four distinct vehicle statuses, which are summarised in Fig. 3(b), also shows an increase in the home parking period $(74.6 \%$ for the weekend) compared with the weekday data.

The probability density function (PDF) of the vehicle driving period per journey for weekdays and weekends, as given in Fig. 4, is dominated by short journeys which are less than half an hour. The 10-minute based TUS data as such can capture vehicle driving patterns in a relatively accurate manner and is therefore beneficial for EV network integration studies; this cannot be achieved by data with lower time resolution.

\section{Time-inhomogeneous Markov Chain Monte Carlo (MCMC) simulation}

The vehicle movement in the TUS data is essentially a series of state transitions throughout a day on a 10-min basis, 
and the transitions between any two different parking locations requires vehicle driving. A Markov Chain diagram as shown in Fig. 5 represents the vehicle state transition from time step $t$ - 1 to $t$, where the four illustrated states, $\{D, H, W$, $C$ \}, correspond to 'driving', 'parking at home', 'parking at workplace', and 'parking at commercial areas' respectively, as identified in the previous section, and the associated transition probability is given for each possible transition at this specific time stamp. For instance, $P_{H \rightarrow D}^{t}$ indicates the probability of the vehicle being ' $\mathrm{D}$ ' at $t$ given being ' $\mathrm{H}$ ' at time $t-1$.

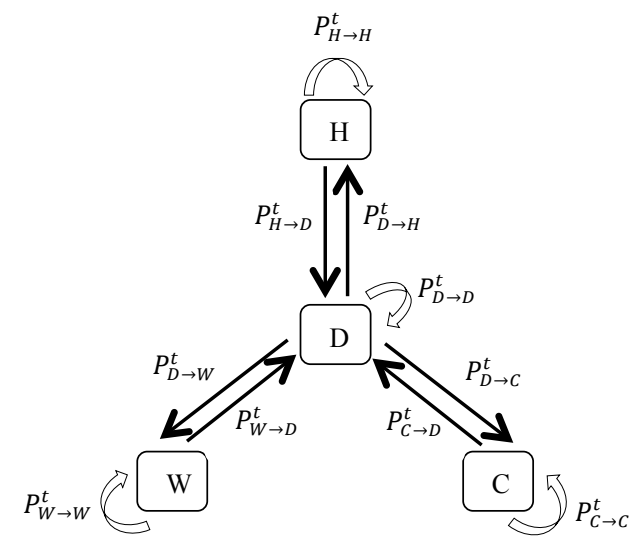

Fig. 5. Markov Chain diagram of possible vehicle state transitions at time $t$ [16]

Fig. 2 exhibits a time-varying feature of vehicle state transition, and to capture such characteristics of vehicle movement the Markov Chain model makes use of time varying transition probabilities as indexed by $t$ in Fig. 5 . Extending the model in this way results in what is known as a time-inhomogeneous Markov Chain, [2]. The simulation period used in this work is 24 hours and includes 144 time stamps, indicating that there will be 143 distinct transition diagrams, i.e. $t \in[2,144]$. The equivalent matrix representation of the transition diagram at time $t, T_{t}$, is expressed by Equation (1), where the transition probabilities from a single state to all the available states are presented in rows, and each row sums to 1 as required for probabilities. Recalling that the transition between any vehicle parking states has to be accomplished via the 'driving' state, the probabilities for infeasible vehicle state transitions without 'driving' are therefore zero.

An example of the state transition matrix at 8:40am $(t=29)$ is shown in Equation (2), which represents a typical weekday morning commuting time. The average trip number for a passenger vehicle per weekday is around 3 according to the TUS data, implying that the state changes would be relatively rare and out of the 144 time stamps the vehicle states will remain constant (in different states) for most of the time, which can be observed by the relatively high probability values of the diagonal elements. The vehicle state transition probability of arrival at work $\left(P_{D \rightarrow W}^{29}\right)$ with a significant conditional probability value of $35 \%$ (of being ' $\mathrm{W}$ ' at $t=29$ given being ' $\mathrm{D}$ ' at time $t=28$ ) is therefore indicative of this being in the morning commuting period.

$$
\begin{aligned}
T_{t} & =\left[\begin{array}{llrr}
P_{D \rightarrow D}^{t} & P_{D \rightarrow H}^{t} & P_{D \rightarrow W}^{t} & P_{D \rightarrow C}^{t} \\
P_{H \rightarrow D}^{t} & P_{H \rightarrow H}^{t} & P_{H \rightarrow W}^{t} & P_{H \rightarrow C}^{t} \\
P_{W \rightarrow D}^{t} & P_{W \rightarrow H}^{t} & P_{W \rightarrow W}^{t} & P_{W \rightarrow C}^{t} \\
P_{C \rightarrow D}^{t} & P_{C \rightarrow H}^{t} & P_{C \rightarrow W}^{t} & P_{C \rightarrow C}^{t}
\end{array}\right] \\
T_{29} & =\left[\begin{array}{rrrr}
0.6172 & 0.0207 & 0.3448 & 0.0172 \\
0.1873 & 0.8127 & 0 & 0 \\
0.0023 & 0 & 0.9977 & 0 \\
0.4444 & 0 & 0 & 0.5556
\end{array}\right]
\end{aligned}
$$

By sampling from the vehicle state PDF as illustrated in Fig. 1(a) and 1(b) at 4am, the initial state at $t=1$ can be determined for weekdays ( 0.0027 for ' $D$ ', 0.9810 for ' $H$ ', 0.0163 for 'W', and 0 for ' $C$ ') and weekend ( 0 for ' $D$ ', 0.9069 for ' $H$ ', 0.0053 for ' $\mathrm{W}$ ' and 0.0878 for ' $\mathrm{C}$ ') respectively, which provides a starting point for sampling from the associated conditional PDF in the state transition matrix as shown in Equation (1). The synthetic driving patterns for the 144 time stamps of a day are then generated based on the time-inhomogeneous Markov Chain matrices, using empirical PDF based Monte Carlo simulation, [18], as the sampling method.

\subsection{Verification of $M C M C$ simulation}

The vehicle driving patterns from MCMC simulation are compared with the TUS data in terms of key statistics for both weekday and weekend driving. To limit repetition only the weekend case is shown in this section.

The average daily (weekend) driving time for passenger cars from the MCMC simulation, as shown by the blue curve in Fig. 6, converges to the TUS value of around 62 minutes as the number of simulation trials increase. This result reflects less driving activity than for weekdays which total on average 80 minutes per day (with similar convergence). It can been seen from Fig. 6 that 10000 trials are sufficient for the simulation to achieve convergence and the subsequent verification results are therefore calculated based on this number of trials. The MCMC simulation based PDF of vehicle driving period per journey and the probability of vehicles' arrival and departure activities, illustrated in Fig. 7 and 8, show slight variations from those from the original TUS data. Despite the small discrepancies for these two statistical measures, the overall vehicle state proportion as calculated from the MCMC simulation agrees very well with the TUS data, as shown in Fig. 9 for weekend data; so does the averaged time for various vehicle states as summarised in Table 2, which matches to one decimal place with the statistics in Fig. 3(b). Similar levels of agreement exist for the weekdays, verifying the capability of MCMC simulation for statistically representative synthetic driving pattern generation.

It is assumed that the driving patterns for EVs in this work share common characteristics with the vehicles in the TUS data, which has been adjusted to take into account the range limitation for EVs. 


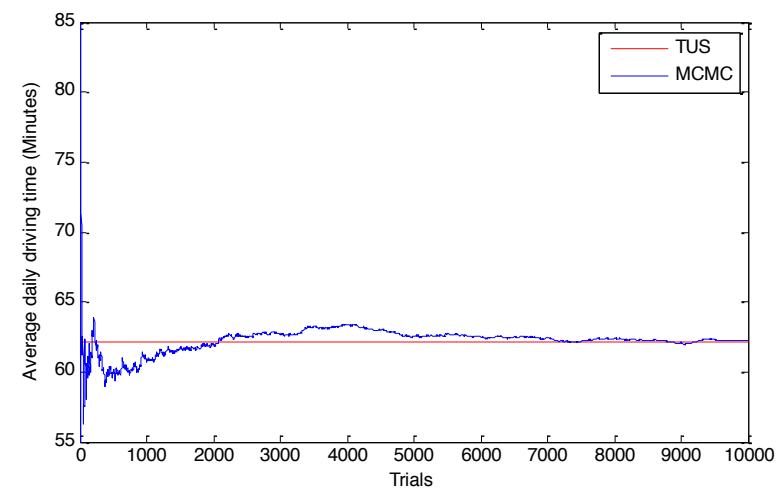

Fig. 6. Average daily driving time convergence for weekend case

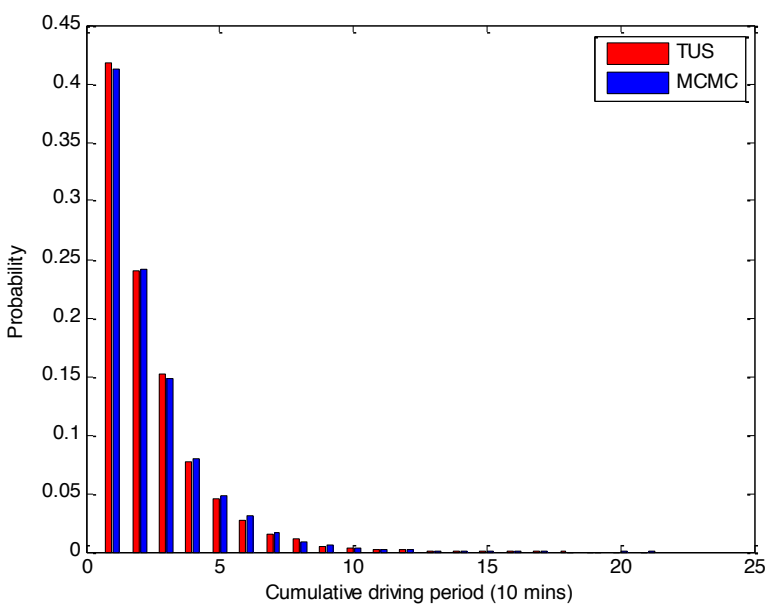

Fig. 7. PDF of vehicle driving period in the weekend
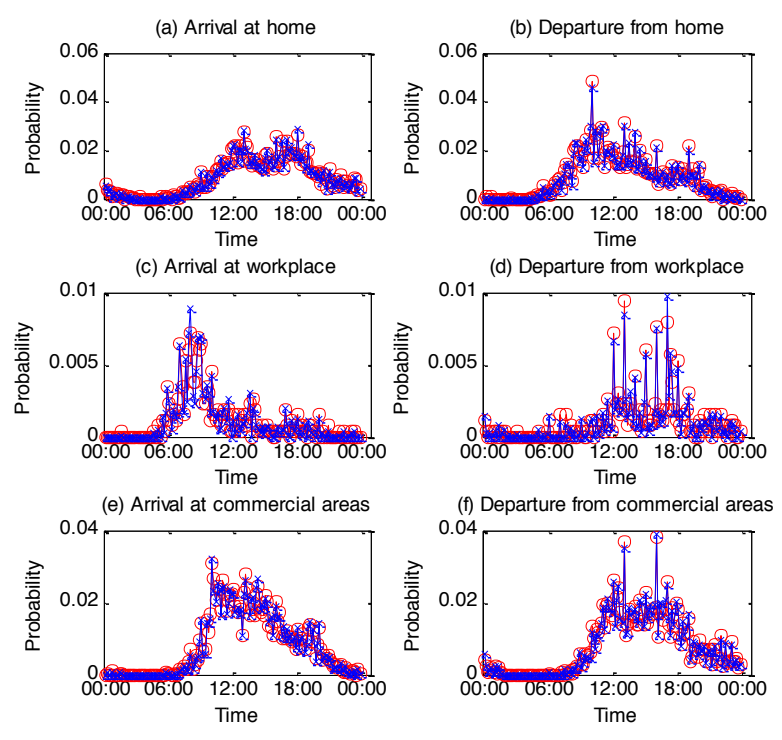

TUS $\longleftarrow$ MCMC

Fig. 8. Probability of vehicles' arrival and departure activities in the weekend
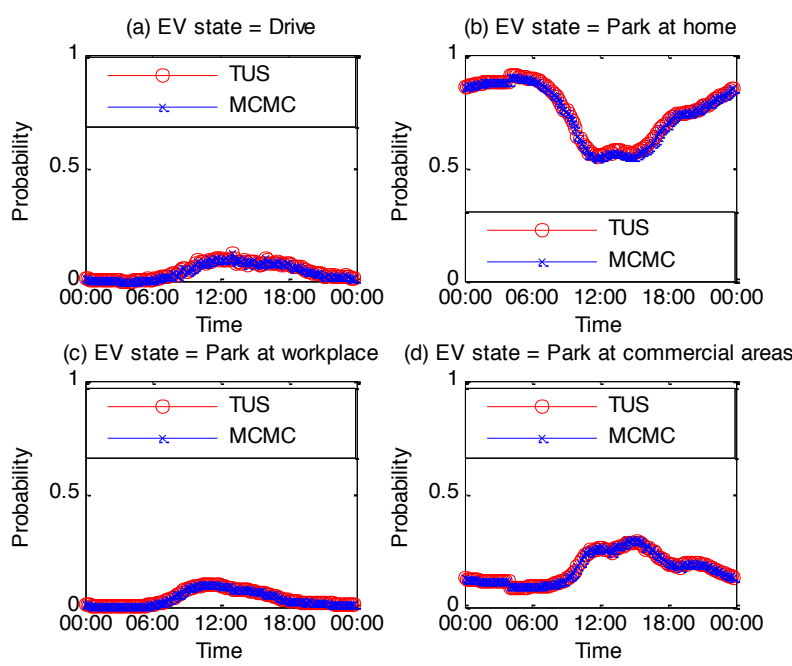

Fig. 9. Vehicle state proportions at the weekend

Table 2

Average time for weekend vehicle states in MCMC simulation results

\begin{tabular}{|l|l|}
\hline \multicolumn{1}{|c|}{ Vehicle state } & Averaged time (hours) \\
\hline Driving & $1.0(4.2 \%)$ \\
\hline Parking at home & $18.0(75 \%)$ \\
\hline Parking at workplace & $0.9(3.7 \%)$ \\
\hline Parking at commercial areas & $4.1(17.1 \%)$ \\
\hline
\end{tabular}

\subsection{Justification of MCMC simulation}

Markov Chain Monte Carlo simulation, as a numerical approach, can be used to generate different electricity load profiles according to various EV charging schemes. The impact of the additional EV charging loads on the local distribution network is assessed by identifying the expected value and associated uncertainty, as measured by the standard deviation, for various grid operational metrics, such as thermal loading, voltage profiles, transformer loss of life, energy losses, and harmonic distortion levels, [19]. And the uncertainty identification of these different metrics requires large number of trials from MCMC simulation to achieve convergence. These uncertainties could not be generated directly by sampling from the original TUS dataset due to its size limitation.

\section{Distribution network case study}

The charging locations of both home and workplace are presented in this work with a 24 hours simulation period, where the initial vehicle battery state of charge is assumed to be $100 \%$ and the battery is returned to a fully charged state by the end of the day to complete the simulation cycle. Based on the daily synthetic EV use patterns generated from the MCMC simulation with 10 minutes step, the EV charging profiles can be calculated assuming uncontrolled charging, where EVs are connected to the grid and charged as soon as they arrive at home. A complete vehicle specification is listed in Table 3 . The two cases with home and workplace charging both take into account the local base load and the additional EV profiles under uncontrolled charging with uncertainties of associated metrics. 
The electric range can be calculated from this specification sheet to be just over 180 minutes, which is used in Section 2 for excessive distance filtering of the TUS data as outlined above.

Table 3

EV specification sheet (taken from BMW i3 model in 2013 [20]) and relevant parameter setting

\begin{tabular}{|l|l|}
\hline Electricity consumption & $12.9 \mathrm{kWh} / 100 \mathrm{~km}$ \\
\hline Averaged driving speed & $30 \mathrm{mph}[21]$ \\
\hline Battery capacity & $18.8 \mathrm{kWh}$ \\
\hline Signle phase standard charging rate & $2.4 \mathrm{~kW}$ \\
\hline Single phase fast charging rate & $7.4 \mathrm{~kW}$ \\
\hline Charging efficiency & $0.9[22]$ \\
\hline Charging load power factor & 1 \\
\hline
\end{tabular}

\subsection{Workplace charging}

A case study of an institutional/commercial building with demand mainly contributed from central heating, lighting, computers, and with typical workplace parking provision is used to assess the direct impact of uncontrolled EV workplace charging on the building load demand as well as the local building supply transformer rating. This example University building at Strathclyde, for which data was available, accommodates up to 300 workers, and has a nominal parking availability for approximately 100 cars. This building is supplied by a dedicated $1000 \mathrm{kVA}$ transformer, and two rates of charging are considered as given in Table 3.

An extreme case of full EV penetration, in this case defined as all vehicles, 100 in total, parking at the workplace being EVs, is studied here, and the aggregate load profiles in apparent power for standard and fast charging cases are shown in Fig. 10 together with the building base demand for a weekday in January, as illustrated by the pink curve. 100,000 simulated EV trials are divided into 1000 groups, each consisting of $100 \mathrm{EVs}$ for this case study. The uncertainty in the aggregate demand, illustrated by the yellow and green shading for the standard and fast charging case respectively, is calculated as the $99 \%$ confidence interval (CI), the upper and lower bound of which is expressed by Equation (3).

$$
\text { 99\%CI bound }=\sqrt{\left(P_{\text {base }}+\mu \pm 2.58 \sigma\right)^{2}+Q_{\text {base }}{ }^{2}}
$$

where $P_{\text {base }}$ and $Q_{\text {base }}$ are the real and reactive power demand for the building; $\mu$ is the mean of the 1000 groups at each time stamp and $\sigma$ is the associated standard deviation; the factor of 2.58 is the Z-table look-up value for $99 \%$ confidence interval based on the assumption of a normal distribution. Fig. 10 illustrates the mean demand profile (in apparent power) of the 1000 groups on top of the building base load for the standard and fast charging cases by solid blue and black lines respectively. Similarly the weekday TUS data, with 1476 diaries, is also grouped into 100 , and the group mean for standard and fast charging rates are illustrated by dotted and dashed lines respectively. The MCMC simulation based mean charging profile shows good agreement with that from the TUS data, which further verifies that the MCMC simulation accurately reflects the TUS data.

Fig. 11 shows an example of the convergence of standard deviation of group charging load (with fast charging rate) at 9:10am, which corresponds to the peak load in the fast charging profile. The mean of the standard deviation gets updated as the group number increases up to group 1000, and the converged value is the $\sigma$ for this time stamp. It can be seen from this figure that the mean of the standard deviation reaches a reasonably stable value from group 300 and the number of EV diaries used for such convergence is significantly higher than that available in the original TUS data, further justifying the use of MCMC simulation.

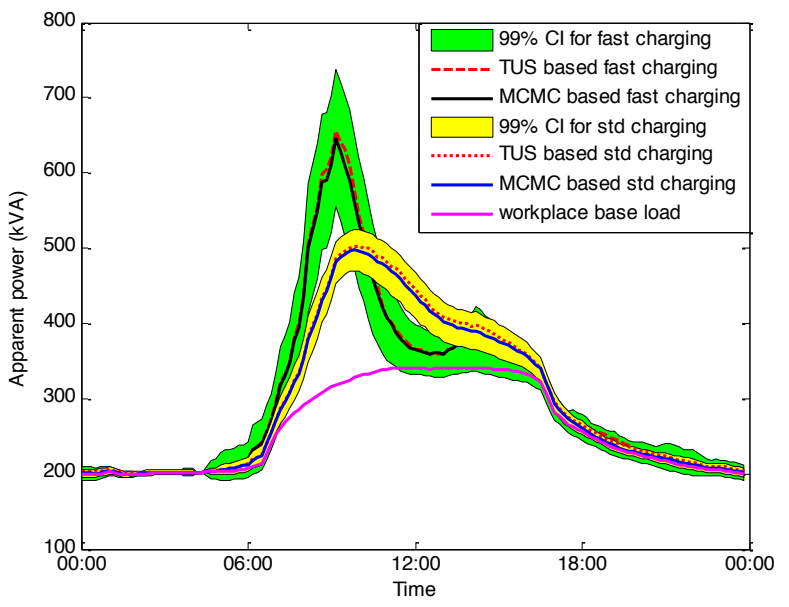

Fig. 10. Aggregate demand of workplace EV charging

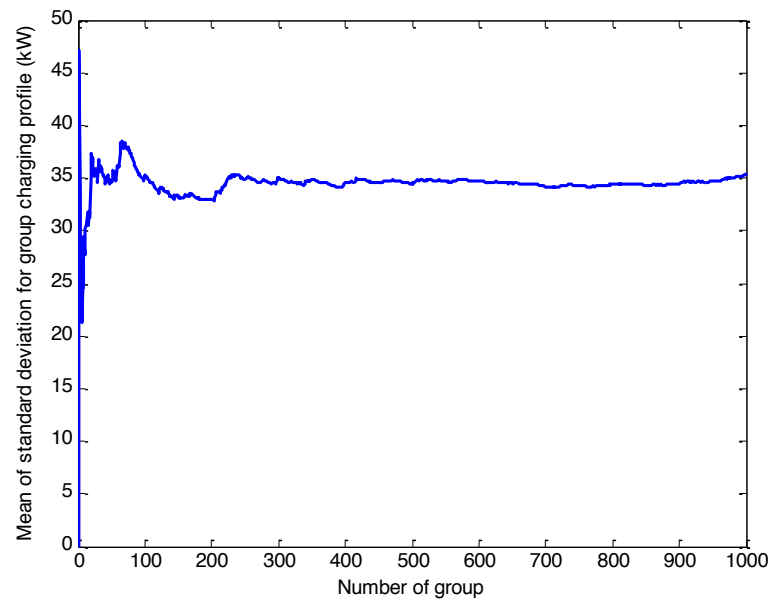

Fig. 11. Convergence of standard deviation of group charging load (with fast charging rate) at 9:10am

In this case of full EV penetration, it can be seen from Fig. 10 that a $1000 \mathrm{kVA}$ transformer would easily survive the extra EV load for both standard and fast charging cases. A more typical transformer for this building with rating of nearer $500 \mathrm{kVA}$ would, however, fail to supply the EV related load in the fast charging scenario, and have some difficulty in the standard charging case. Such events could cause degradation of building supply transformer and also indicate the potential for adverse impact on power quality for the users in the building as a result of excess demand. For future planning of the local network that involves EV charging, transformer sizing should be undertaken with consideration of the 
aggregate load together with its associated uncertainty, i.e. there should be sufficient positive margin between the transformer size and the upper bound of the confidence interval for aggregate demand.

\subsection{Home charging}

Impact of uncontrolled EV charging on the local distribution network, in terms of the household voltage profiles and the thermal characteristic of the substation feeder and the associated uncertainty, is investigated under the standard charging rate for both weekday and weekend cases. Fig. 12 shows the layout of the investigated low-voltage single-phase domestic network that consists of 17 households, which is extracted from the three-phase distribution system as structured in [23] with slightly modified network attributes.

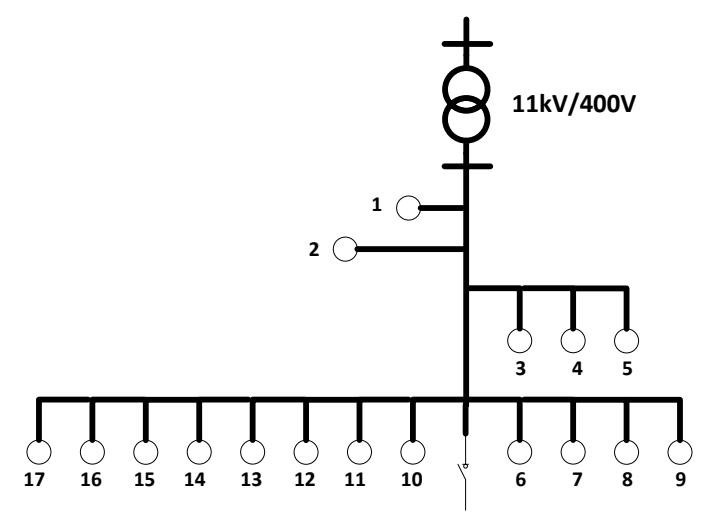

Fig. 12. Single phase distribution network layout

A domestic electricity consumption model, [15], also developed based on TUS data, is employed here to generate the load profile for domestic households alongside the EV charging profiles. The input of this model includes a series of parameters, such as day of week, month of the year and active occupancies. A January weekday and weekend are chosen as input for this model to represent the typical peak winter profile, and a power factor of 0.9 is assumed for the domestic loads; an occupancy number that is randomly chosen from 1 to 5 is allocated to each of the simulated dwellings. Household 17 that is located at the farthest end of the branch is investigated here as it is subject to the lowest voltages. The generated demand profiles of a weekday and at the weekend for this household are shown in Fig. 13 by the blue and red curves respectively, together with the associated $\mathrm{EV}$ charging profiles that are generated according to the energy requirement and EV availability, and an assumption that the vehicles start charging immediately on return to the house.

The voltage profile for Household 17, which suffers the most from the network impact due to its location, is investigated under the full EV penetration scenario for both a weekday and at the weekend. As used in the workplace charging case, 1000 groups are chosen here, for each of which the EV profiles are generated for the 17 households with an individually modelled base demand. A dedicated network simulator, Open Distribution System Simulator (OpenDSS) [24], is employed here to undertake the power flow analysis.

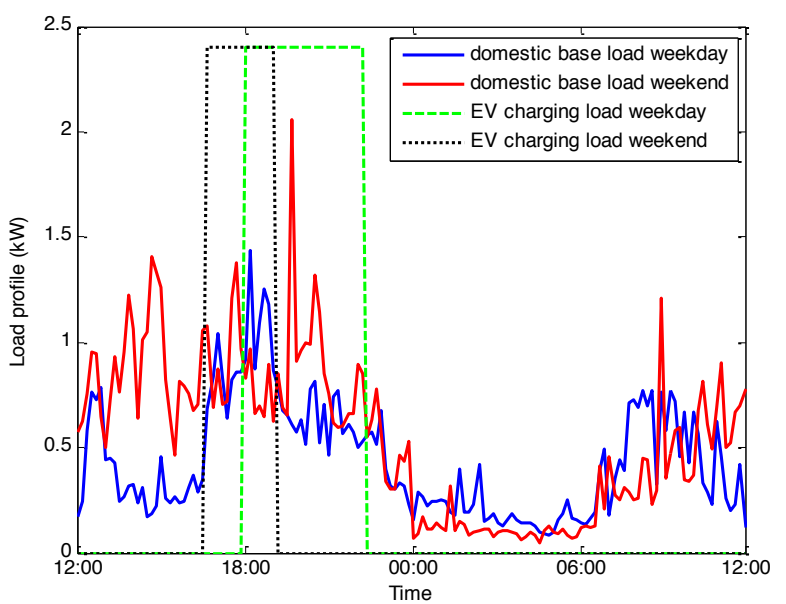

Fig. 13. Example of domestic base load and the charging profile for Household 17

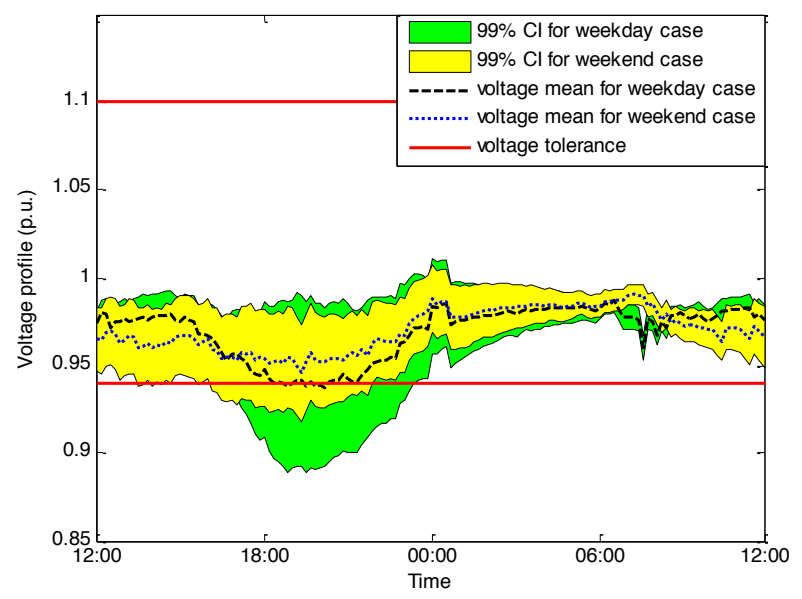

Fig. 14. Averaged voltage profile for Household 17 with $99 \%$ CI under full EV penetrations

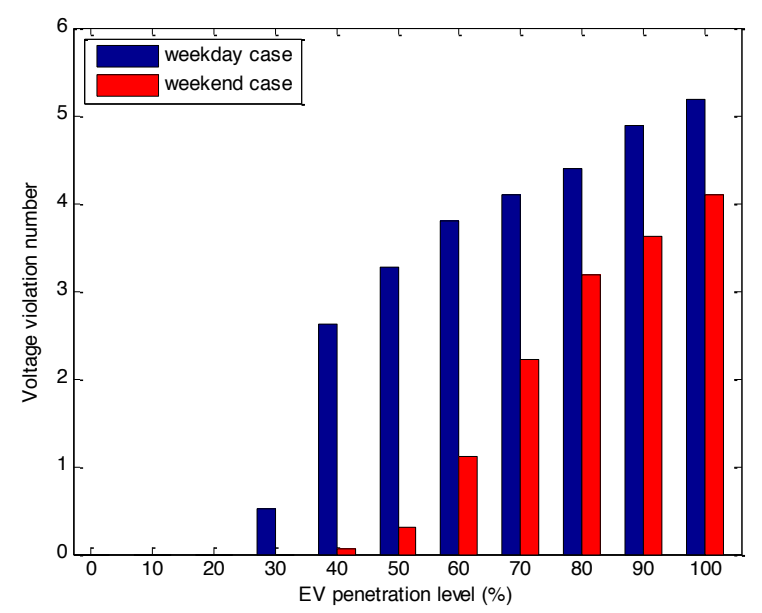

Fig. 15. Averaged hours of lower voltage bound (of $99 \% \mathrm{CI}$ ) excursion under different EV penetrations

The converged voltage profiles of the 1000 groups are presented in Fig. 14 for Household 17 giving mean voltage values and the associated $99 \%$ CI. It can be seen from this 
figure that the extreme EV penetration in this case causes a severe voltage violation of the network (with specified tolerance of [-0.06 +1.10] p.u., [25]) during the peak hours for both weekday and weekend cases, with the former showing a much higher uncertainty range than the latter. In general the weekday voltage profile shows a lower trend than that for the weekend, which is due to the longer driving period for weekdays, as discussed in Section 2, and therefore a greater charging energy requirement. Also the majority of people arrive home between $4 \mathrm{pm}$ and $9 \mathrm{pm}$ for weekdays, as is shown in Fig. 2(a), and uncontrolled charging scenario would coincide with the residential base demand peak thus worsening the network issues. It is worth pointing out that even though the mean weekday voltage level is within the acceptable voltage range, its uncertainty extends to a voltage value well below the threshold and therefore implies a significant probability of being unacceptable. The representation of uncertainty is essential to network analysis and a $99 \%$ confidence interval as adopted here provides a reasonable level of risk reduction regarding the voltage profile.

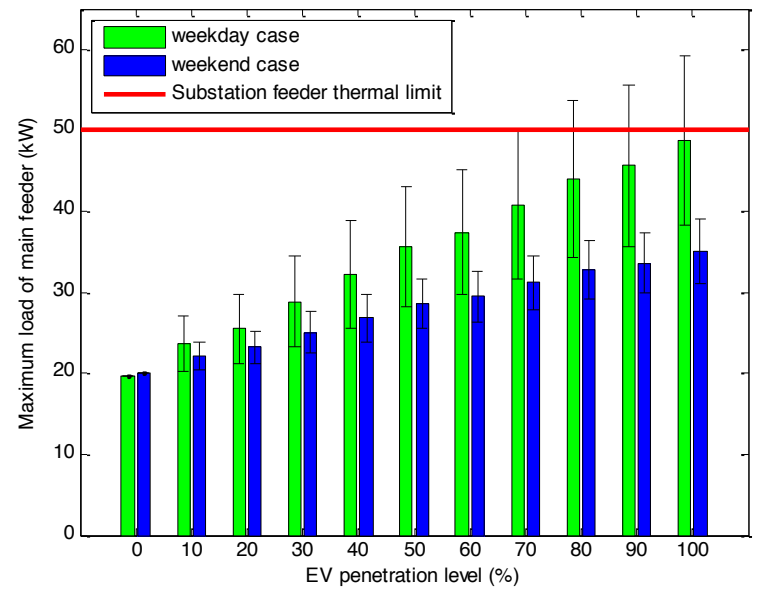

Fig. 16. Substation feeder thermal characteristics with $99 \%$ CI

A further investigation of total voltage violation number for the entire households is carried out under different EV penetrations, where one EV per household is assumed for the households that are selected to be equipped with EVs. Fig. 15 summarises the excursions of the lower voltage bound ( $99 \%$ CI) in terms of averaged hours per household. Over 5 and 4 hours of voltage excursion per household is observed for weekdays and the weekend at an EV $100 \%$ penetration, which indicates a similar degree of network issues for the entire households as for Household 17 shown in Fig. 14 where the excursion period is around 6 and 5 hours for weekdays and weekends respectively. The weekday case can be observed to place more stress on the network than the weekend throughout all the EV uptake levels and the $99 \%$ CI indicates a network tolerance level for EV penetration of up to $20 \%$ for weekdays and $30 \%$ for the weekend.

One of the solutions to voltage violation is to adjust the substation transformer tap setting, which typically ranges within $5 \%$ either side of the nominal ratio in $2.5 \%$ or $5 \%$ steps
[25], to maintain the voltage even at the furthest end of a feeder within the required tolerances. In this case with a heavy EV charging demand, a $+2.5 \%$ transformer tapping would alleviate the voltage burdens on the network to some extent.

The thermal performance of the substation feeder as shown in Fig. 16 exhibits a similar trend to Fig. 15, with error bars representing the uncertainty. It can be seen in Fig. 16 that for both the weekday and weekend case the uncertainty range increases as the EV penetration level rises. The specified line thermal limit, $50 \mathrm{~kW}$ in this case, allows the weekend to survive under all penetrations but could only just accommodate up to a $70 \%$ penetration for weekdays.

\section{Conclusions and future research}

The Markov Chain Monte Carlo simulation has been verified to preserve the statistical features of vehicle use patterns from the TUS data and therefore is suitable for use in network integration analyses. The case studies for workplace and home charging show different degrees of network stress in terms of various operational metrics due to uncontrolled vehicle charging, and the $99 \%$ confidence interval that is obtained from the MCMC simulation results provides a statistically significant representation of the network impact uncertainties, which is important for system planning and operation. Future works will explore smart charging methods with potential vehicle to grid actions under a smart grid environment to mitigate system operational issues and avoid unnecessary cost of network reinforcement.

\section{Acknowledgements}

This work was funded by the Engineering and Physical Sciences Research Council under Grant EP/K005316/1 and $\mathrm{EP} / \mathrm{L} 01004 / 01$.

\section{References}

[1] T.-K. Lee, Z. Bareket, T. Gordon, and Z. S. Filipi, "Stochastic Modeling for Studies of Real-World PHEV Usage: Driving Schedule and Daily Temporal Distributions," IEEE Trans. Veh. Technol., vol. 61, no. 4, pp. 1493-1502, May 2012.

[2] F. J. Soares, J. P. Lopes, P. R. Almeida, C. L. Moreira, and L. Seca, “A stochastic model to simulate electric vehicles motion and quantify the energy required from the grid", PSCC, Stockholm, Sweden, 2011.

[3] E. B. Iversen, J. K. Møller, J. M. Morales, H. Madsen, "Inhomogeneous Markov models for describing driving patterns", IEEE Transactions on Power Systems.

[4] A. Lojowska, D. Kurowicka, G. Papaefthymiou, and L. van der Sluis, "Stochastic modeling of power demand due to EVs using copula", IEEE Transactions on Power Systems, vol. 27, no. 4, pp. 1960-1968, 2012.

[5] A. Ashtari, E. Bibeau, S. Shahidinejad, and T. Molinski, "PEV charging profile prediction and analysis based on vehicle usage data." IEEE Trans. Smart Grid, vol. 3, no. 1, pp. 341-350, 2012.

[6] A. D. Hilshey, P. D. Hines, P. Rezaei, and J. R. Dowds, "Estimating the impact of electric vehicle smart charging on distribution transformer aging." IEEE Trans. Smart Grid vol. 4, no. 2, pp. 905-913, 2013.

[7] F. Rassaei, W. S. Soh, and K. C. Chua, "Demand response for residential electric vehicles with random usage patterns in smart grids." IEEE Transactions on Sustainable Energy vol. 6, no. 4, pp, 1367-1376, 2015.

[8] M. Fotuhi-Firuzabad, M. Rastegar, A. Safdarian, and F. Aminifar, "Probabilistic home load controlling considering plug-in hybrid electric vehicle uncertainties." in Reliability Modeling and Analysis of Smart Power Systems. Springer India, 2014, pp. 117-132. 
[9] A. H. Mohsenian-Rad and M. Ghamkhari, "Optimal charging of electric vehicles with uncertain departure times: A closed-form solution", IEEE Trans. Smart Grid, vol. 6, no. 2, pp. 940-942, 2015.

[10] J. Fluhr, K. H. Ahlert, and C. Weinhardt, "A stochastic model for simulating the availability of electric vehicles for services to the power grid." In System Sciences (HICSS), 43rd Hawaii International Conference on. IEEE. 2010, pp. 1-10.

[11] J. D. Melo, E. M. Carreno, and A. Padilha-Feltrin, "A multi-agent system with a percolation approach to simulate the driving pattern of Plug-In ELectric Vehicles," in 2012 IEEE PES Transmission and Distribution Conference and Exposition (T\&D), 2012, pp. 1-7.

[12] S. Shafiee, M. Fotuhi-Firuzabad, and M. Rastegar, "Investigating the impacts of plug-in hybrid electric vehicles on power distribution systems." IEEE Transactions on Smart Grid, vol. 4, no. 3, pp. 1351-1360, 2013.

[13] National Household Travel Survey, [Online]. Available: http://nhts.ornl.gov/index.shtml

[14] MobiliteitsonderzoekNederland (MON), Databestand MON, 2008.

[15] I. Richardson, M. Thomson, D. Infield, and C. Clifford, "Domestic electricity use: A high-resolution energy demand model," Energy and Buildings, vol. 42, no. 10, pp. 1878-1887, Oct. 2010.

[16] Y. Wang, S. Huang, and D. Infield, "Investigation of the potential for electric vehicles to support the domestic peak load." In Electric Vehicle Conference (IEVC), IEEE. Dec. 2014, pp. 1-8.

[17] "The United Kingdom 2000 Time Use Survey," National Statistics Technical Report, 2003.
[18] R.Y. Rubinstein and D.P. Kroese, "Random number, random variable, and stochastic process generation," in Simulation and the Monte Carlo method, John Wiley \& Sons, 2011.

[19] R. C. Green, L. Wang, and M. Alam, "The impact of plug-in hybrid electric vehicles on distribution networks: A review and outlook." Renewable and Sustainable Energy Reviews, vol. 15, no. 1, pp. 544-553, 2011.

[20] BMW i3 technical data. [Oline] Available: http://www.bmw.com/com/en/newvehicles/i/i3/2016/showroom/technica 1 data.html

[21] Department for Transport (DfT), Transport Statistics: Great Britain 2009 Edition 35th Edition ed.: TSO (The Stationery Office), 2009.

[22] W. Kempton and J. Tomić, "Vehicle-to-grid power fundamentals: calculating capacity and net revenue", Journal of power sources, vol. 144, no. 1, pp. 268-279, 2005.

[23] S. Huang, "Modelling of the impact of electric vehicle take-up on the power distribution network", Ph.D. dissertation, Depart. Electronic and Electrical Engineering, University of Strathclyde, Glasgow, UK, 2014.

[24] The Open Distribution System Simulator (OpenDSS), Electric Power Research Institute, Inc., June 2016. [Online] Available: https://sourceforge.net/projects/electricdss/

[25] Department of Trade and Industry (DTI), "The electricity safety, quality and continuity regulations", Stationary Office, London, UK, Oct. 2002.

[26] E. Lakervi and E J. Holmes "Network Voltage Performance," in Electricity distribution network design, No. 21. IET, 1995. 